\title{
CORRECTION
}

\section{Correction to: AdaStereo: An Efficient Domain-Adaptive Stereo Matching Approach}

\author{
Xiao Song ${ }^{1} \cdot$ Guorun Yang ${ }^{2} \cdot$ Xinge $\mathrm{Zhu}^{3} \cdot$ Hui Zhou $^{1} \cdot$ Yuexin $\mathrm{Ma}^{4} \cdot$ Zhe Wang $^{1} \cdot$ Jianping Shi $^{1}$
}

Accepted: 24 January 2022 / Published online: 9 February 2022

(c) Springer Science+Business Media, LLC, part of Springer Nature 2022

\section{Correction to: \\ International Journal of Computer Vision https://doi.org/10.1007/s11263-021-01549-6}

The article AdaStereo: An Efficient Domain-Adaptive Stereo Matching Approach written by Xiao Song - Guorun Yang · Xinge Zhu · Hui Zhou · Yuexin Ma · Zhe Wang · Jianping

The original article can be found online at https://doi.org/10.1007/ s11263-021-01549-6.

Xiao Song

songxiao@sensetime.com

Guorun Yang

yangguorun91@gmail.com

Xinge Zhu

zhuxinge123@gmail.com

Hui Zhou

zhouhui@sensetime.com

Yuexin Ma

mayuexin@shanghaitech.edu.cn

Zhe Wang

wangzhe@ sensetime.com

Jianping Shi

shijianping@sensetime.com

1 Sense Time Group Limited, Hong Kong, China

2 Shenzhen Institutes of Advanced Technology, Chinese Academy of Sciences, Beijing, China

3 Chinese University of Hong Kong, Hong Kong, China

4 Shanghai Tech University, Shanghai, China
Shi was originally published electronically on the publisher's Internet portal (currently SpringerLink) on 01 January 2022 with open access. With the author(s)' decision to step back from Open Choice, the copyright of the article changed on [24/01/2022] to (OThe Author(s), under exclusive license to The Indian Econometric Society 2022, and the article is forthwith distributed under the terms of copyright.

Publisher's Note Springer Nature remains neutral with regard to jurisdictional claims in published maps and institutional affiliations. 